\title{
Estimation of hydromechanical parameters of limb lymphedematous tissue with the use of chamber tests
}

\author{
MARIUSZ KACZMAREK ${ }^{1 *}$, JOANNA NOWAK ${ }^{1}$, \\ WALDEMAR L. OLSZEWSKI ${ }^{2}$, MARZANNA ZALESKA ${ }^{2}$ \\ ${ }^{1}$ Department of Mechatronics, Kazimierz Wielki University, Bydgoszcz, Poland. \\ ${ }^{2}$ Medical Research Center, Polish Academy of Sciences, Warsaw, Poland.
}

\begin{abstract}
Purpose: In this paper, in vivo methods of estimation of the shear modulus and hydraulic permeability of subcutaneous tissue of lower limb are presented. Methods: The experimental technique is based on single- or two-chamber inflation-deflation tests in which temporal changes in limb circumference under the test chamber for cyclic loading are registered. Simplified models for fast undrained deformation and slow creep of oedematous tissue with squeezing out interstitial liquid were considered. Finite element simulations of the chamber test within a finite deformation poroelastic model were elaborated. Results: Formulas necessary to estimate the shear modulus and permeability of subcutaneous tissue were derived and then tested or calibrated using the results of poroelastic simulations. An example of application of the derived formulas for clinical data obtained from the chamber test was discussed. Conclusions: A simple in vivo methods of estimation of the hydromechanical properties of lymphedematous tissue (shear modulus and permeability) were proposed. The strengths and weaknesses of the proposed methodology were discussed.
\end{abstract}

Key words: soft oedematous tissue, chamber test, estimation, elastic parameters, permeability, large strain poroelasticity

\section{Introduction}

Lymphedema is understood as a set of pathologic conditions characterized by the regional accumulation of excessive amounts of interstitial protein-rich fluid [19]. Lymphedema of the lower limbs is usually a consequence of obstruction of the main lymphatic vessels due to inflammation, trauma, excision of lymph nodes or irradiation. Patients with lymphoedema have chronic changes in limb size that may have a substantial effect on their quality of life. Thus, diagnosis of the presence or stage of lymphedema is needed to establish treatment for patients suffering from this disease.

Among the therapies available for oedema reduction (rehabilitative interventions, pharmacological therapies, and surgery), one of the most promising methods is intermittent pneumatic compression (IPC) [7], [22], [23]. This conservative method is based on mechani- cal interaction with tissue in a multichamber system that is sequentially inflated and deflated. Appropriate pressures and time periods of inflation and deflation of the IPC device should be determined to obtain an optimal decrease in limb volume. The parameters of the IPC device must be adjusted with respect to hydromechanical tissue characteristics. This adjustment is even more significant for the rational application of more complex therapeutic modalities involving a combination of bandaging, stockings and IPC and for monitoring the effects of such treatments.

Considering that lymphedematous tissue is a two-phase material composed of tissue matrix filled with interstitial fluid [20], its fundamental tissue characteristics are parameters of elasticity and permeability. The elasticity of tissue determines reversible tissue deformation, whereas permeability is crucial for predicting the intensity of interstitial fluid outflow from the tissue due to compression. Simple and reliable

\footnotetext{
* Corresponding author: Mariusz Kaczmarek, Department of Mechatronics, Kazimierz Wielki University, ul. Kopernika 1, 85-074 Bydgoszcz, Poland. Phone: +48 52 3257650, fax:+48 523257644, e-mail: mkk@ukw.edu.pl

Received: October 9th, 2020

Accepted for publication: January 21st, 2021
} 
clinical methods that can be used to identify oedematous tissue properties to help select optimum IPC parameters are required.

Most noninvasive techniques applied to study the mechanical properties of soft tissues are based on indentation or air flow (suction or blow) methods [2], [6], [11], [12], [18], [24] or MRI and ultrasonic elastography methods [1], [27]. Then, the tissue properties are determined using the experimental results and appropriate mechanical models of tested limb sections. The drawback of such techniques used for lymphedematous or adipose limb tissues, however, is that they are applied on a relatively small domain of tissue, and, as a result, the determined properties are valid locally. Moreover, local deformations of the skin are then significant, and, as a result, the skin may play an equally important role as a subcutaneous tissue in the test, and separation of their contributions in the test is not straightforward. An alternative clinical technique that has been recently developed to determine optimum IPC parameters is the two-chamber inflationdeflation method [23]. The important features of this technique are that tissue loading is applied on a relatively large surface area, which guarantees the identification of representative mechanical characteristics of spatially inhomogeneous subcutaneous tissue and that the role of skin becomes less important than it is in the indentation test. Moreover, the conditions resemble the procedure used in IPC, and, therefore, the same apparatus could be used for diagnostic checks and treatment in the future. The limitation of the method presented in [23] results from the fact that it concentrates on the qualitative analysis of mechanical tissue responses due to cyclic loading, particularly on residual tissue deformation after unloading, both under and next to the chamber. As a result, the method does not deliver quantitative tissue characteristics, such as elastic parameters or permeability, which are clinically desired for proper parameter adjustment of compression therapy.

The aim of this paper was to present methods developed to estimate the elastic shear modulus and hydraulic permeability of subcutaneous tissue based on single- or two-chamber inflation-deflation tests. The aforementioned method [23], designed to acquire data on changes in limb circumference, was applied. Cyclic loading of tissue with increasing maximum chamber pressures in consecutive cycles was assumed in order to ensure at least one cycle in which the two stages of tissue deformation, fast undrained deformation and slow creep of the drained matrix, were present. Analytical models for the finite deformation problem of solid material in the first stage and for squeezing out interstitial fluid from permeable material in the second stage were proposed. Formulas for the estimates of shear elasticity parameter and permeability of subcutaneous tissue were obtained. The methods were tested or calibrated using results from simulations based on direct integration of a continual poroelastic model, and an example of their application for clinical data was discussed.

\section{Methods}

The proposed method refers to the experimental method known as the chamber tests and uses simplified modelling of tissue deformation and flow of fluid from the compressed tissue in order to derive appropriate analytical formulas. 3D finite element simulations for poroelastic tissue model were performed to complement the analytical results.

\subsection{Experimental method}

Two similar techniques using a single or double chamber inflation-deflation test as schematically shown in Fig. 1 were considered. The individual chambers had the same width $(9 \mathrm{~cm})$ and were placed around a limb. In both cases, the test chamber was inflated sequentially to $50,80,100$, and optionally $120 \mathrm{mmHg}$ for 1-3 minutes at each pressure. The deflation periods between the loadings lasted for approximately 1 minute. In the double chamber test, the chambers were mechanically connected but pressurized independently. The chamber preventing backflow of tissue fluid was placed above the ankle joint and inflated to $120 \mathrm{mmHg}$, and then, the test chamber started to be inflated. The loadings were transmitted to the tissue, and changes in limb circumference at the control point located in the middle of the contact with the test chamber were recorded using plethysmographic strain gauges. In the original two-chamber technique proposed in [23], the circumference was also measured a few centimetres proximal to the test chamber, but in the methods considered here, those results were not used.

An example of the results obtained from a two-chamber inflation-deflation test for a patient diagnosed with lymphedema is shown in Fig. 2. Three pressure levels $(50,80$, and $100 \mathrm{mmHg})$ were applied for 3 minutes with a 1-minute pause between the loads. The periods of inflation and deflation lasted for approximately $5 \mathrm{~s}$ at each loading level. The curve represents changes in leg circumference in the central cross section of the chamber. 
a)

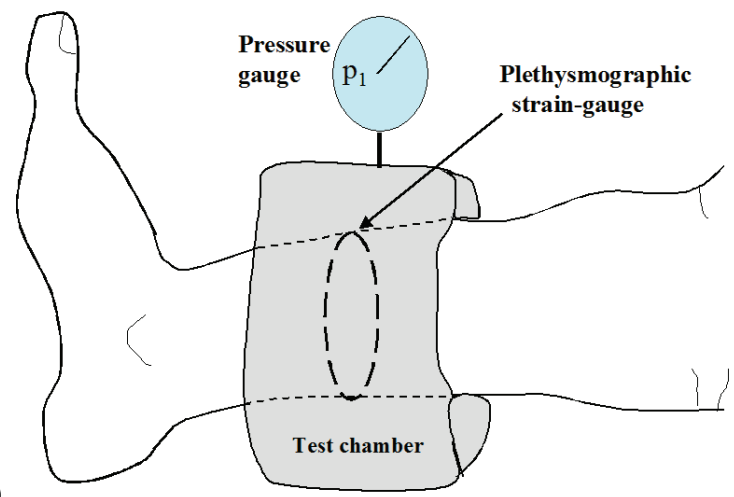

b)

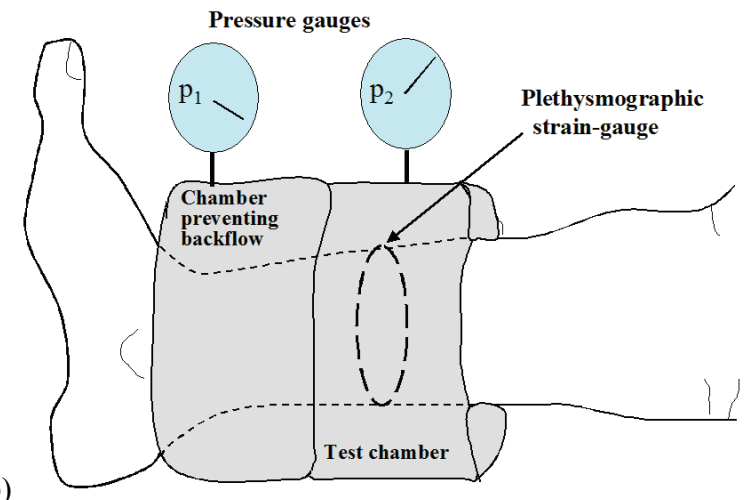

Fig. 1. Single (a) and two-chamber (b) inflation-deflation tests

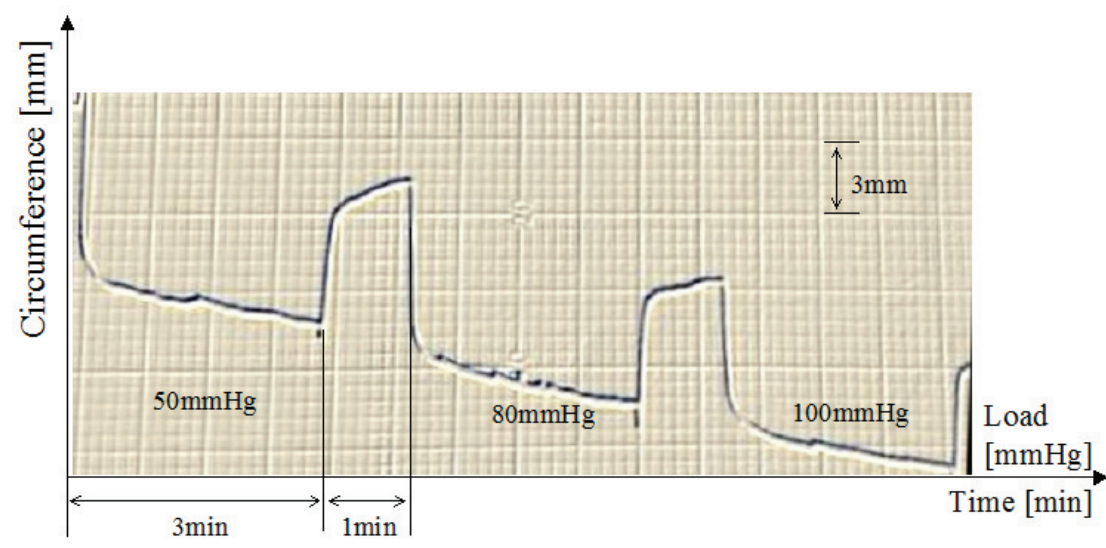

Fig. 2. Example of two-chamber test results for patients with a diagnosis of lymphedema showing circumference changes versus time for three loading levels

For each loading, first, a relatively fast deformation process (change of circumference) occurred, and then, a period of much slower deformation of the loaded part of the leg was observed. According to the idea that soft oedematous tissue should be treated as a solid matrix filled with interstitial fluid, the first (fast) part of the process reflects undrained tissue deformation, which is mostly displaced perpendicularly to the loading direction. The second part of the process is related to tissue deformation due to drainage or squeezing of interstitial fluid from the tissue com- bined with the recurred motion of the matrix as a result of a difference in the undrained and drained Poisson's ratio.

\subsection{Tube model of undrained tissue deformation}

The mechanical tube model of soft oedematous limb tissue was considered (Fig. 3) in the framework of a large deformation range [26]. We assumed that

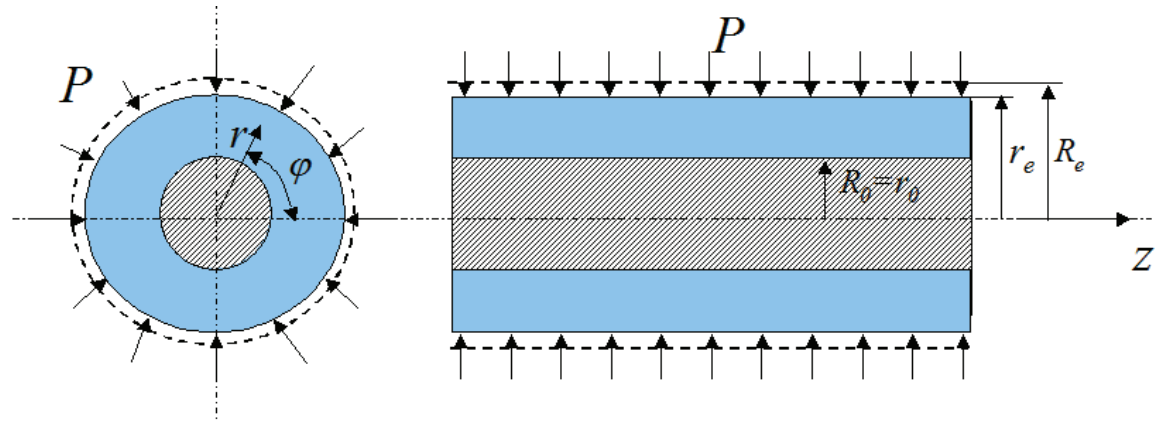

Fig. 3. Tube model of limbs under loading by pressure $P$ in the first stage of the chamber test 
the layer of soft oedematous tissue has constant thickness and that the cylindrical core (bone and muscles) is undeformable, tissue stiffness and axial stretch are homogeneous, and skin stiffness is disregarded. The tissue was modelled as an incompressible neo-Hookean elastic material neglecting the relative displacement of its fluid component (undrained deformation). The latter assumption was justified by the fact that the tissue permeability was low enough that in a short loading period, deformation occurred much faster than the outflow of fluid. Assuming that the load was applied as pressure $P$ on the outer surface of the limb model and disregarding any tangential interaction between the oedematous layer and underlying tissues or with the surface of the chamber, the stress can be treated as independent of the $z$ axis. The resulting problem became a plane problem in that the solution for stress depends only on the radial coordinate.

Adopting geometrical and physical assumptions introduced in Section 2.2, we considered the tube model of undrained soft oedematous limb tissue. In view of the conditions of incompressibility of undrained tissue and homogeneous axial deformations, we obtained

$$
\lambda_{r} \lambda_{\varphi} \lambda_{z}=1
$$

where

$$
\lambda_{r}=\frac{d r}{d R}, \quad \lambda_{\varphi}=\frac{r}{R}, \quad \lambda_{z}=\chi
$$

are the radial, circumferential and axial stretch ratios, respectively; $\chi$ is a constant; $R$ and $r$ denote the radial coordinates in the initial and deformed states, respectively. Combining (1) and (2), we got

$$
\frac{d r}{d R} \frac{r}{R} \chi=1,
$$

which integrated gave

$$
R=\sqrt{\chi r^{2}+C},
$$

where the integration constant $C$ can be found from the condition that for $r=r_{0}$, we have $R=R_{0}=r_{0}$ and then $C=(1-\chi) r_{0}^{2}$. Then, from (4) for $R=R_{e}$, the axial stretch ratio is

$$
\chi=\frac{R_{e}^{2}-r_{0}^{2}}{r_{e}^{2}-r_{0}^{2}} .
$$

The constitutive relationships for the diagonal components of the Cauchy stress tensor $\sigma_{r r}, \sigma_{\varphi \varphi}$, and $\sigma_{z z}$ for neo-Hookean material are as follows [5]

$$
\begin{aligned}
\sigma_{r r} & =\mu B_{r r}-p, \\
\sigma_{\varphi \varphi} & =\mu B_{\varphi \varphi}-p, \\
\sigma_{z z} & =\mu B_{z z}-p,
\end{aligned}
$$

where $B_{r r}, B_{\varphi \varphi}, B_{z z}$ are the components of the left Cauchy-Green deformation tensor, $\mu$ is the shear modulus, and $p$ denotes the constitutively undetermined pressure. Expressing the components of $\boldsymbol{B}$ by stretches and using (2) and (4), they read

$$
\begin{gathered}
B_{r r}=\lambda_{r}^{2}=\frac{\chi r^{2}+C}{\chi^{2} r^{2}}, \quad B_{\varphi \varphi}=\lambda_{\varphi}^{2}=\frac{r^{2}}{\chi r^{2}+C}, \\
B_{z z}=\chi^{2} .
\end{gathered}
$$

The equilibrium equation in the radial direction was

$$
\frac{\partial \sigma_{r r}}{\partial r}+\frac{1}{r}\left(\sigma_{r r}-\sigma_{\varphi \varphi}\right)=0 .
$$

Then, substituting (6) and (7) into (8), the differential equation for pressure $p$ was obtained, which integrated over the radial coordinate gave

$$
p=\mu\left[\frac{C}{2 \chi^{2} r^{2}}+\frac{\ln (r)}{\chi}-\frac{\ln \left(\chi r^{2}+C\right)}{2 \chi}\right]+D
$$

and the integration constant $D$ determined from the boundary condition $r=r_{e} \Rightarrow \sigma_{r r}=-P$ read $D=\mu\left[\frac{1}{\chi}+\frac{C}{2 \chi^{2} r_{0}^{2}}+\frac{\ln \left(\chi r_{0}^{2}+C\right)}{2 \chi}-\frac{\ln \left(r_{0}\right)}{\chi}\right]+P$.

Two conditions for equilibrium of the considered tissue element in the axial direction were considered. The first condition required that the resultant axial force must be equal to zero

$$
\int_{r_{0}}^{r_{e}} 2 \pi r \sigma_{z z} d r=0
$$

and the alternative condition required that for the outer boundary, the axial stress vanishes

$$
\sigma_{z z}\left(r=r_{e}\right)=0 .
$$

The analysis presented above refers to both single- and two-chamber tests. In the single chamber test, the displacements occur symmetrically in both directions from the middle cross-section, whereas in the two-chamber test, there is no displacement at the contact boundary with the chamber preventing fluid backflow, and as a result, there are only proximal displacements. 


\subsection{Model of squeezing out interstitial fluid from tissue}

The tissue deformed in the first - undrained - stage of the loading underwent slow drainage. Considering that the time scale of the second stage is much longer than that of the first stage and that the tissue is permeable, we modelled the drained deformations as the process when the interstitial fluid is squeezed out from the loaded to the unloaded part of the layer of tissue, neglecting explicit coupling of the fluid flow with deformation of tissue matrix (Fig. 4). For the same porous material, the undrained Poisson's ratio is larger than the drained Poisson's ratio. Therefore, volumetric changes in the tissue must be balanced by the volume of fluid squeezed out and the volume of the matrix material that recurs at the same time.

The following set of assumptions was postulated to build a simple model of the process. The axial flow of interstitial fluid was solely considered. The material of the solid matrix and the pore fluid were assumed to be incompressible. The maximum fluid pressure located at the origin of the $z$ coordinate remained constant throughout the second stage of the test. The complex distribution of deformation close to the edge of the chamber and the deformation of tissue in the unloaded part were not considered. The length of the considered section of the limb was assumed to be such that for $z=B+b$, the pore pressure was dissipated. $B$ was equal to half of the chamber width for the single chamber test and was identical to the chamber width for the two-chamber test.

Following the introduced model assumptions (Section 2.3) and considering first the loaded part of the limb, we can write the balance between the change in tissue volume $V(z)$ in the closed limb section from 0 to $z \leq B$ and the volumetric flow rate of tissue $J(z)$

$$
\frac{\partial V(z)}{\partial t}=J(z)
$$

The instantaneous change in volume $V(z)$ can be expressed by the product of the lateral surface area of the cylinder $2 \pi r_{e} z$ and the average radial velocity of the outer boundary $v=\frac{d r_{e}}{d t}$, i.e.,

$$
\frac{\partial V(z)}{\partial t}=2 \pi r_{e} z V
$$

The volumetric flow rate of tissue $J(z)$ has two components. One component is related to the volumetric flux of the squeezed-out fluid $\phi v^{f}$, and the other is related to the volumetric flux of the matrix material $(1-\phi) v^{s}$, where $\phi$ denotes porosity and $v^{f}$ and $v^{s}$ are the average velocities of pore fluid and solid matrix, respectively. The latter volumetric flux appears because in the drained deformation, the matrix recurs, partially replacing the squeezed-out fluid (the Poisson's ratio of the undrained porous material is larger than that of the drained material). The fluxes have opposite directions, and since globally, the circumference within the chamber decreases, the fluid flux prevails. Considering the above, it can be postulated that the volumetric flow rate $J(z)$ is proportional to the volumetric flux of pore fluid with respect to solid $q(z)=\phi\left(v^{f}-v^{s}\right)$ multiplied by the surface area of the cross section of tissue

$$
J(z)=-m \pi\left(r_{e}^{2}-r_{0}^{2}\right) q(z),
$$

where $m$ is a proportionality constant. The assumed approximation for $J(z)$ means that the velocity of the recurred matrix $v^{s}$ can be expressed as $v^{s}=(m-1) q$, from which $m$ has a value between 0 and 1 .

Supposing that the velocity $v$ is independent of time and space (axial coordinate $z$ ) and combining Eqs. (12)-(14), we obtained the following:

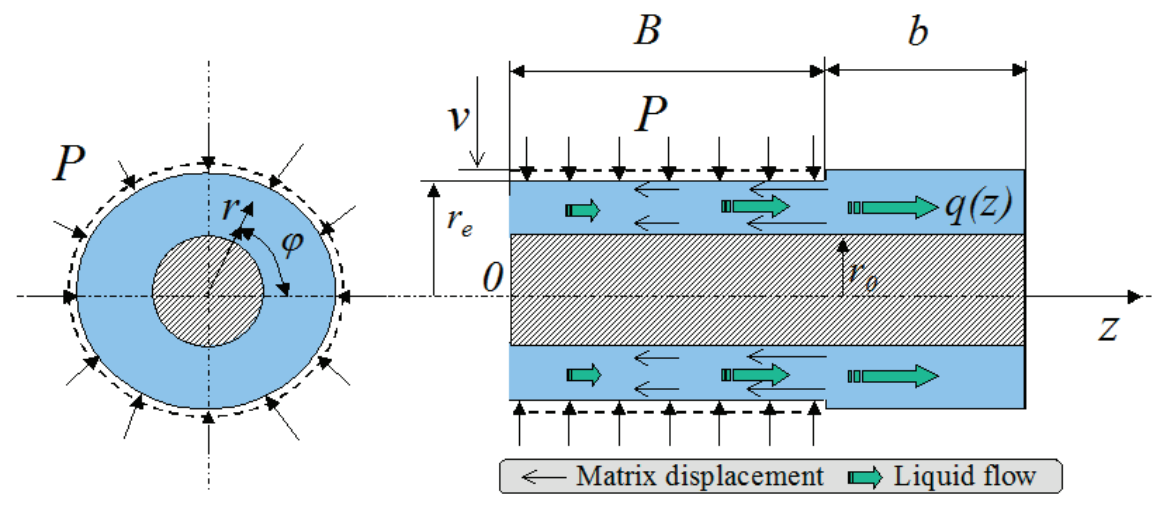

Fig. 4. Model of outflow of fluid and recurring matrix for the second stage of the chamber test 


$$
q(z)=-\frac{2 r_{e} z v}{m\left(r_{e}^{2}-r_{0}^{2}\right)} .
$$

The flow of interstitial fluid can be described by Darcy's equation, which relates the gradient of the average pore fluid pressure $\bar{p}(z)$ and discharge velocity $q(z)$

$$
\frac{d \bar{p}(z)}{d z}=-\frac{\eta}{k} q(z)
$$

where $\eta$ is the fluid viscosity and $k$ represents permeability.

By substituting (16) into (15), one obtains the differential equation for pressure as a function of $z$, and this equation can be integrated from 0 to $B$, giving the following

$$
\bar{p}_{B}=\bar{p}_{0}+\frac{\eta r_{e} v}{m k\left(r_{e}^{2}-r_{0}^{2}\right)} B^{2}
$$

where $\bar{p}_{0}=\bar{p}(0)$ and $\bar{p}_{B}=\bar{p}(B)$.

Considering the assumption that pressure vanishes for $z=B+b$ and using Darcy's equation (16) for the unloaded part of the limb model (from $z=B$ to $z=B+b$ ), we got

$$
\frac{\bar{p}_{B}}{b}=\frac{\eta}{k} q_{B}
$$

where $q_{B}=q(B)$, and from equation (15), we received the following

$$
q_{B}=-\frac{2 r_{e} B v}{m\left(r_{e}^{2}-r_{0}^{2}\right)} .
$$

Parameters $r_{0}, r_{e}, B$ and $b$ should be adjusted on a case-by-case basis depending on the geometry of the limb and on which test (single or double) is considered.

\subsection{Poroelastic simulations of tissue in the two-chamber test}

The lymphedematous tissue is composed of mutually interacting tissue matrix and interstitial fluid [13], [20]. Simulations of oedematous tissue loaded by a single compression chamber using the small strain poroelastic model were reported in [13]. Here, the model was extended for finite deformation and the two-chamber test. The mathematical model applied here was based on finite deformation poroelasticity, [15], which assumes the principle of effective stress and a neo-Hookean model of a solid skeleton. Moreover, the isotropy and homogeneity of the tissue and no variation of material parameters (mechanical stiffness, porosity and permeability) were postulated. The adopted geometry and constraints on the displacement of tissue are shown in Fig. 5.

The flow of interstitial fluid across the boundary is possible only for the free boundary $z=L$. The assumed loading history is the same as applied in the clinical test (loadings of 50, 80 and $100 \mathrm{mmHg}$ over $180 \mathrm{~s}$ with

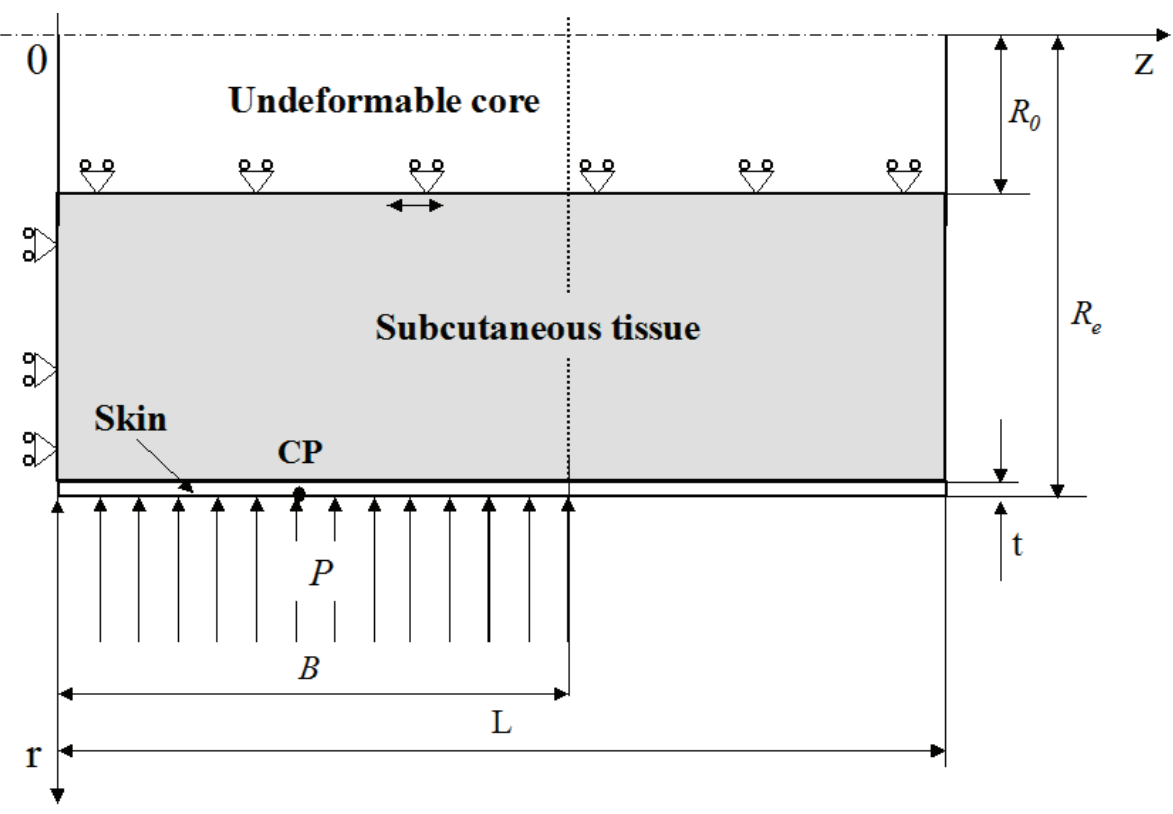

Fig. 5. Geometry of the modelled limb section with loading (pressure $P$ ).

The control point $(\mathrm{CP})$ is located in the middle of the loaded section 
Table 1. Parameters assumed for simulations

\begin{tabular}{|l|l|l|}
\hline \multicolumn{1}{|c|}{ Skin } & \multicolumn{1}{|c|}{ Oedematous tissue } & \multicolumn{1}{c|}{ Geometry } \\
\hline Thickness, $t=1 \mathrm{~mm}$ & Drained Young's modulus, $E_{m}=12.5 / 25 / 50 \mathrm{kPa}$ & $L=B+b=120 \mathrm{~mm}$ \\
\hline \multirow{2}{*}{ Young's modulus, $E_{s}=0.25 / 0.5 \mathrm{MPa}$} & Drained Poisson's ratio, $v_{m}=0.33$ & $B=90 \mathrm{~mm}$ \\
\cline { 2 - 3 } & Mass density, $\rho=1000 \mathrm{~kg} / \mathrm{m}^{3}$ & $R_{0}=60 \mathrm{~mm}$ \\
\hline \multirow{2}{*}{ Poisson's ratio, $v_{s}=0.49$} & Permeability, $k=5 / 15 / 45 \times 10^{-14} \mathrm{~m}^{2}$ & $R_{e}=90 \mathrm{~mm}$ \\
\cline { 2 - 3 } & Viscosity, $\eta=0.001$ Pas & \\
\hline \multirow{2}{*}{ Mass density, $\rho_{s}=1000 \mathrm{~kg} / \mathrm{m}^{3}$} & Porosity, $\phi=0.05$ & \\
\cline { 2 - 3 } & Effective stress coupling, $B=1$ & \\
\hline
\end{tabular}

a 5 -s increase and 60 -s break between loadings). The parameters adopted for performing simulations are given in Table 1.

The Young's modulus for skin can range from $10^{4}$ to $10^{7} \mathrm{~Pa}$, and for subcutaneous tissue, it can range from $10^{2}$ to $10^{5} \mathrm{~Pa}$ [4], [10], [16]. In our simulations, we considered moderate values of the parameter: for skin, $E_{s}=0.25$ and $0.5 \mathrm{MPa}$, and for subcutaneous tissue, $E_{m}=12.5,25$ and $50 \mathrm{kPa}$. Young's modulus for subcutaneous tissue refers to drained conditions. The Poisson's ratio used in modelling skin is usually close to 0.5 , and we assumed $v_{s}=0.49$.

Due to the lack of data for the drained Poisson's ratio of subcutaneous tissue with oedema, we assumed its value as for the brain tissue [14], i.e., $v_{m}=0.33$. Given the assumed values of drained Young's modulus and Poisson's ratio, we found, using the formula for the shear modulus $\mu=0.5 \mathrm{E} /(1+v)$, that the considered values of shear modulus of subcutaneous tissue (identical for drained and undrained conditions) are then $\mu_{m}=4.7,9.4$ and $18.8 \mathrm{kPa}$. According to the results of tissue microscopic studies, [3], the percentage of free (mobile) liquid in subcutaneous tissue in relation to the whole liquid is small, and we assumed $\phi=0.05$. The permeability of animal subcutaneous tissue without oedema and with artificially induced oedema can range from $10^{-16}$ to $10^{-9} \mathrm{~m}^{2}$ [9], and we considered the values $k=5,15$ and $45 \times 10^{-14} \mathrm{~m}^{2}$. The effective stress coefficient $B$ was assumed to be equal to unity, and the parameters describing the interstitial fluid, density $\left(\rho_{f}\right)$, compressibility $\left(K_{f}\right)$, and viscosity $(\eta)$ were taken as those for water.

The simulations were performed based on the finite element method implemented in the COMSOL Multiphysics environment (version 4.2a). The model was implemented with the help of Solid Mechanics and Darcy's law Modules assuming axial symmetry. Convergence in a reasonable time and solution stability were ensured using a mesh with 4494 triangular elements, and the maximum and minimum element sizes were $5.55 \mathrm{~mm}$ and $0.0188 \mathrm{~mm}$, respectively.

\section{Results}

The analytical formulas for estimation of parameters of subcutaneous tissue from chamber tests were derived based on models introduced in Sections 2.2 and 2.3. The results of 3D poroelastic simulations were used to justify the simplifications made for deriving the analytical models, validate the method for estimation of the shear modulus and to calibrate the unknown parameter in the equation for permeability.

\subsection{Formulas for estimating shear modulus and permeability of tissue}

In order to find formulas for shear modulus of tissue $\mu$ from the tube model of undrained tissue, we used equations (6), (7) and (9) and considered the equilibrium conditions (10) or (11). As a result we received:

$$
\begin{gathered}
\mu=P\left[2 \chi^{2} r_{e}^{2}\left(r_{e}^{2}-r_{e}^{2}\right)\right]\left[2 r_{e}^{4} \chi^{4}+r_{e}^{2} \ln \left(\chi r_{e}^{2}+C\right)\right. \\
-2 r_{e}^{2} C \ln \left(r_{e}\right)-2 r_{0}^{2} r_{e}^{2} \chi^{4}-r_{0}^{2} r_{e}^{2} \chi \ln \left(\chi r_{0}^{2}+C\right) \\
=r_{e}^{2} C \ln \left(\chi r_{0}^{2}+C\right)+2 r_{0}^{2} r_{e}^{2} \chi \ln \left(r_{0}\right)+2 r_{e}^{2} C \ln \left(r_{0}\right) \\
-2 r_{e}^{4} \chi+2 r_{0}^{2} r_{e}^{2} \chi-C\left(r_{e}^{2}-r_{0}^{2}\right)+r_{0}^{2} r_{e}^{2} \chi \ln \left(\chi r_{e}^{2}+C\right) \\
\left.-2 r_{0}^{2} r_{e}^{2} \chi \ln \left(r_{e}\right)\right]^{-1}
\end{gathered}
$$

or

$$
\mu=P\left[\chi^{2}-\frac{1}{\chi}-\frac{C}{\chi^{2} r_{e}^{2}}\right]^{-1} .
$$

Equations (20) or (21) can be used to calculate the shear modulus of subcutaneous tissue. It is worth adding that, according to the poro-elastic model, the value of the shear modulus determined in conditions without drainage was the same as the value of the shear value with drainage. 
The permeability of compressed tissue can be derived from the model of squeezing out interstitial fluid from tissue combining equations (17)-(19) from which we got

$$
k=-\frac{\eta r_{e} v B(B+2 b)}{M P\left(r_{e}^{2}-r_{0}^{2}\right)},
$$

where the product $m \bar{p}_{0}$ was replaced by the product of the chamber pressure $P$ and a new parameter $M$ that required calibration.

\subsection{Results of poroelastic simulations}

In order to justify the simplifications made for deriving the analytical models of undrained deformation of tissue and squeezing of interstitial fluid from tissue, in Fig. 6, examples of distributions of radial displace

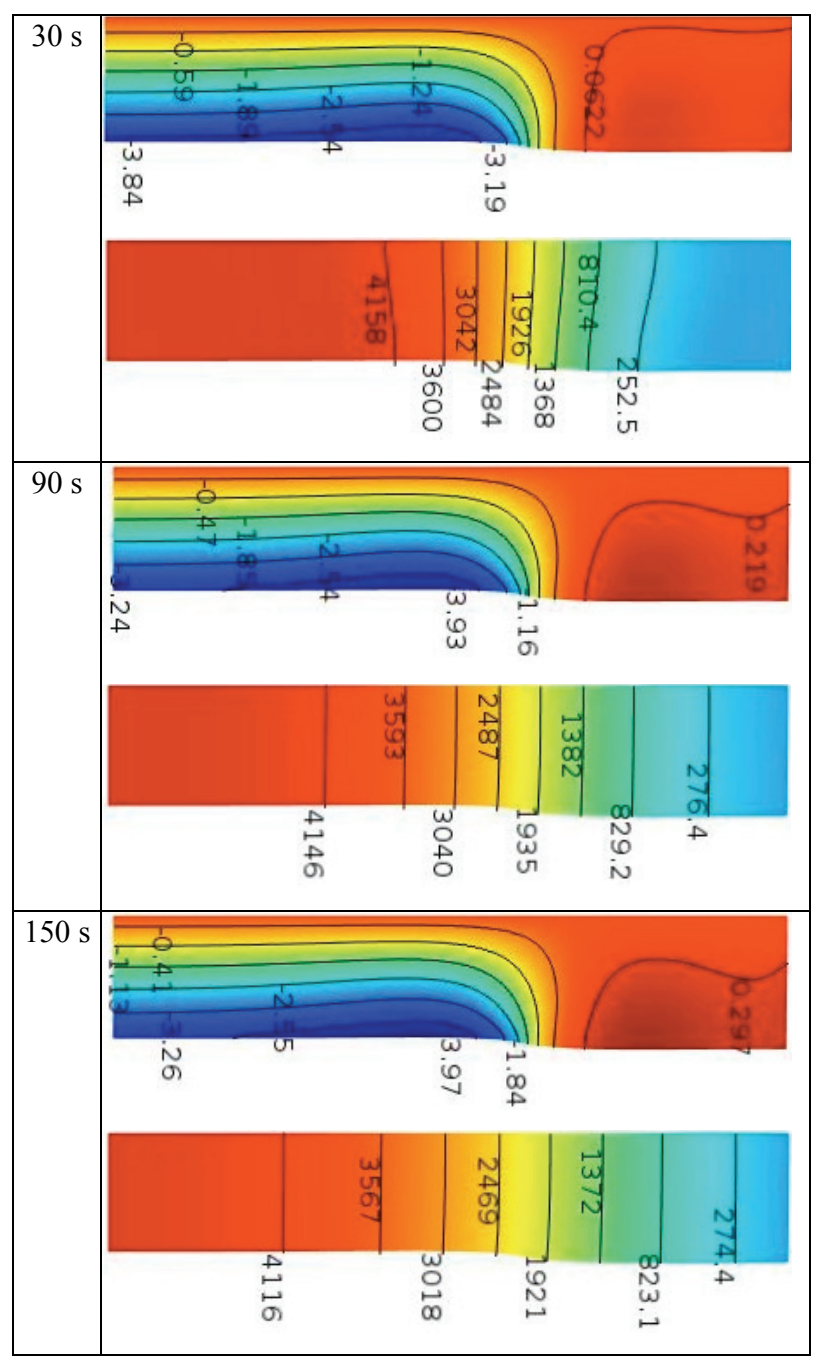

Fig. 6. Transient distributions of radial displacement of the solid matrix (top map, labels are given in $\mathrm{mm}$ ) and interstitial fluid pressure (bottom map, labels are given in $\mathrm{Pa}$ ) predicted by FE simulations for observation times of 30,90 and $150 \mathrm{~s}$ and a load of $50 \mathrm{mmHg}$ ment of the solid skeleton (the top map for a given time, labels in $\mathrm{mm}$ ) and pore pressure (the bottom map for a given time, labels in $\mathrm{Pa}$ ) for the first period of loading (50 mmHg) and time points of 30, 90 and $150 \mathrm{~s}$ were shown. The results involve the same crosssection shown in Fig. 5 and material characteristics: $E_{s}=0.25 \mathrm{MPa}, E_{m}=25 \mathrm{kPa}$ and $k=15 \times 10^{-14} \mathrm{~m}^{2}$.

Excluding the domain close to the chamber's edge, the radial components of tissue displacement within the chamber are only slightly dependent on $z$-axis and show slow systematic increases both within the chamber (negative values) and next to it (positive values). The isolines of pore pressure within the chamber are parallel and roughly perpendicular to the $z$-axis. The shapes of the isolines for radial displacement and pressure justify the simplifications made for deriving the analytical models of undrained deformation of tissue (Section 2.2) and squeezing of interstitial fluid from tissue (Section 2.3).
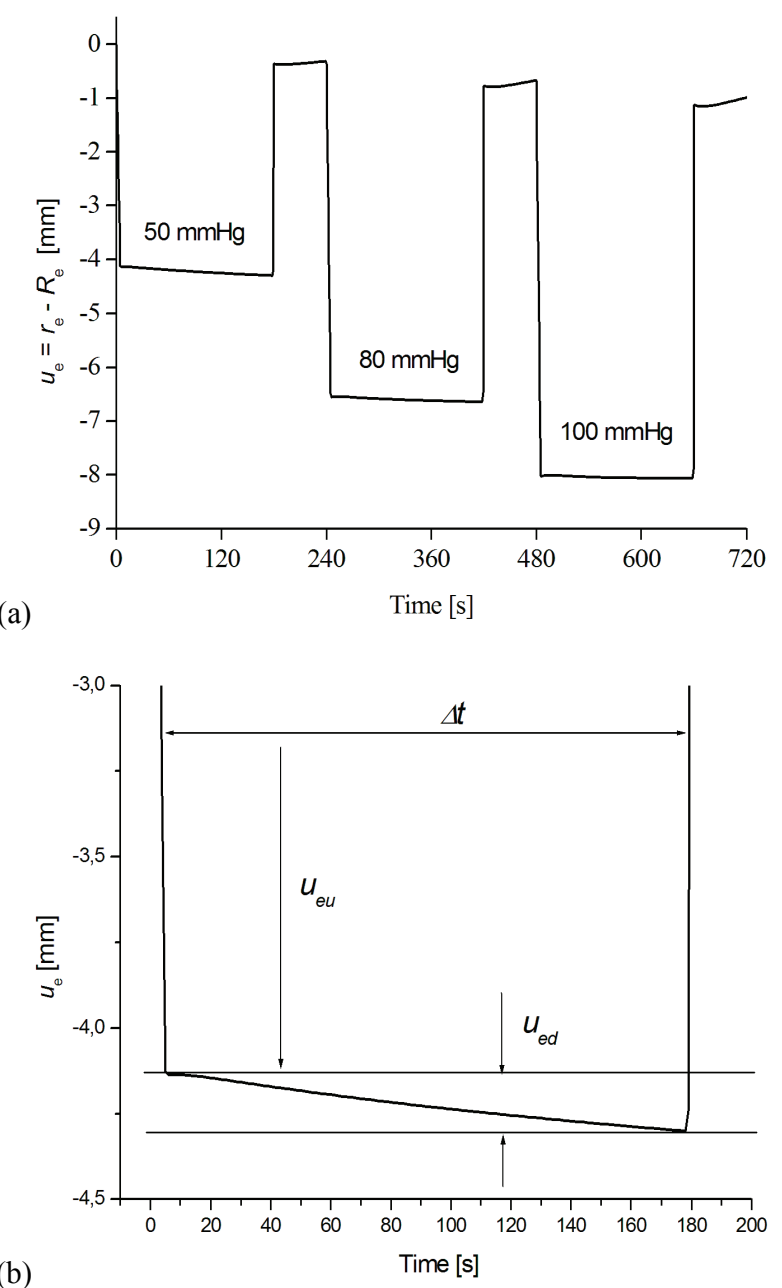

Fig. 7. Radial displacement of the control point $(\mathrm{CP})$ due to loads of 50,80 , and $100 \mathrm{mmHg}$ (a) and division into undrained $u_{e u}$ and drained $u_{e d}$ displacements for the first load cycle (b) 
The time dependence of the radial displacement $u_{e}=r_{e}-R_{e}$ of the control point (CP) for the same data as used above is shown in Fig. 7a. For a single cycle, the total displacement can be divided into displacement in the initial stage $u_{e u}$ - without drainage and displacement in the second stage $u_{e d}$, which occurs under drainage conditions (Fig. 7b). The radial velocity of the point $\mathrm{CP}$ in the second stage can be defined as $v=u_{e d} / \Delta t$, where the time increment $\Delta t$ amounts to 3 minutes.

The numerical results showing axial displacements of the matrix just beneath the proximal edge of the test chamber are presented in Fig. 8 to support the analysis presented in Section 2.3 concerning the components of volumetric flux of fluid in the second (drained) stage of deformations. For each load period, a large initial deformation is followed by slow matrix recurrence (motion in the opposite direction). It should be stressed out that on one hand, the latter effect enhances tissue drainage, which is mostly driven by the interstitial fluid pressure gradient; however, on the other hand, it reduces the volumetric changes of loaded tissue.

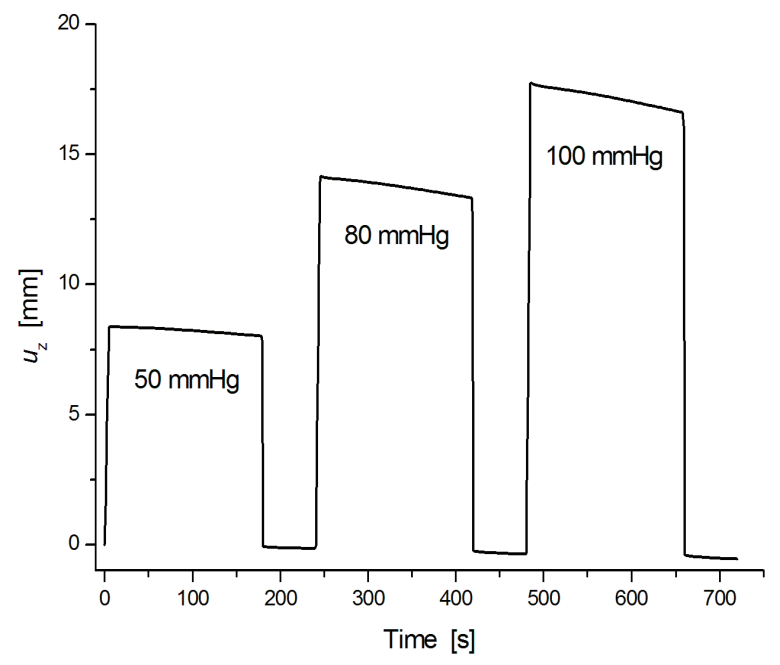

Fig. 8. Axial tissue displacement beneath the proximal edge of the test chamber

\subsection{Validation of the formula for tissue shear modulus}

The shear modulus of undrained tissue can be estimated using formula (20) or (21). The value of radius $r_{e}=R_{e}+u_{e}$ corresponding to the undrained deformation of the outer boundary can be determined by reading displacement $u_{e u}$ from the results of simulations (Fig. 7b) for a given geometry, material parameters and loading cycle of the limb. In Table 2, the values of the estimated moduli for each of the three load periods with the original value of the shear modulus used in the simulations when $E_{s}=0.25 \mathrm{MPa}$, $E_{m}=25 \mathrm{kPa}, v_{m}=0.33$ and $k=15 \times 10^{-14} \mathrm{~m}^{2}$ are compared. It is worth noting that, regardless of the applied condition for determining the shear modulus (zero axial force or vanishing axial stress at the outer boundary), the obtained results are close to each other and at higher loads approached the input modulus. The difference between the values of the shear modulus predicted by equations (20) and (21) and the value taken for the simulation amounts to a maximum of approximately $10 \%$ for the data from the first load cycle and decreases in subsequent cycles. An important reason for the occurrence of such a difference is the fact that when deriving formulas (20) and (21), the skin stiffness was omitted. In turn, the decrease in the difference for the subsequent cycles is a consequence of the residual deformations caused by the delay in the outflow of liquid from the tissue and the interference of this effect with the elastic response.

For a more detailed assessment of the effects of omitting the skin in the tissue stiffness modulus estimation model, the results of 3D poroelastic simulations and formula (21) were used for all considered combinations of stiffness of subcutaneous tissue and skin, and the results are summarized in Table 3. It can be seen that the greater the difference in the stiffness of the subcutaneous tissue and the skin, the greater the estimation error in relation to the value taken for the simulation. It should be noted, however, that from

Table 2. Comparison of estimated values of the shear modulus determined from equations (20) and (21) using the results of simulations for different loading levels

\begin{tabular}{|c|c|c|c|c|}
\hline \multirow{2}{*}{$\begin{array}{c}\text { Shear modulus } \\
\text { used in simulations } \\
\text { (input data) } \\
{[\mathrm{kPa}]}\end{array}$} & \multicolumn{4}{|c|}{ Estimated shear modulus for different loadings [kPa] } \\
\cline { 2 - 5 } & Equation & $\begin{array}{c}1 \mathrm{st} \text { cycle } \\
50 \mathrm{mmHg} \\
\left(u_{e u}=4.1 \mathrm{~mm}\right)\end{array}$ & $\begin{array}{c}\text { 2nd cycle } \\
80 \mathrm{mmHg} \\
\left(u_{e u}=6.2 \mathrm{~mm}\right)\end{array}$ & $\begin{array}{c}\text { 3rd cycle } \\
100 \mathrm{mmHg} \\
\left(u_{e u}=7.6 \mathrm{~mm}\right)\end{array}$ \\
\hline \multirow{2}{*}{9.4} & $(20)$ & 10.34 & 10.3 & 9.57 \\
\cline { 2 - 5 } & $(21)$ & 10.37 & 10.06 & 9.959 \\
\hline
\end{tabular}


a physiological point of view, it is unlikely, even in pathological conditions, that low stiffness of the subcutaneous tissue may be accompanied by high stiffness of the skin. A similar analysis performed for different permeabilities showed that this parameter did not have a major impact on the estimation of the shear modulus, which confirms the validity of the assumption of the undrained nature of the deformation in the first load stage.

Table 3. Estimated values of the shear modulus using the results of simulations for different stiffnesses of subcutaneous tissue and skin (results for load $50 \mathrm{mmHg}$ )

\begin{tabular}{|c|c|c|c|}
\hline & \multicolumn{3}{|c|}{$\begin{array}{l}\text { Shear modulus of subcutaneous } \\
\text { tissue used in simulations* }[\mathrm{kPa}]\end{array}$} \\
\hline & 4.7 & 9.4 & 18.8 \\
\hline $\begin{array}{c}\text { Young's modulus of skin } \\
\text { Es used in simulations } \\
{[\mathrm{MPa}]}\end{array}$ & \multicolumn{3}{|c|}{$\begin{array}{l}\text { Shear modulus estimated } \\
\text { from equation }(21)[\mathrm{kPa}]\end{array}$} \\
\hline 0.25 & 5.5 & 10.3 & 19.5 \\
\hline 0.5 & 7.6 & 12.5 & 21.8 \\
\hline
\end{tabular}

* The given shear moduli were calculated from the relation $\mu=0.5 E /(1+v)$ taking the values of Young's modulus $E=12.5$, 25 and $50 \mathrm{kPa}$ and Poisson's ratio $v=0.33$ into account.

\subsection{Calibration of the model to estimate permeability}

Effective application of Eq. (22) to estimate the permeability of subcutaneous tissue from a chamber test required finding the parameter $M$, and this was be the aim of this calibration. Because the derivation of Eq. (22) was based on the postulate of linear pore fluid transport, the procedure was used solely for the first period of loading. Given the 3D poroelastic simulations of the limb for the selected geometrical and material parameters, the drained component of the radial displacement $u_{e d}$ (see Fig. $7 \mathrm{~b}$ ) cumulated over 3 minutes $(\Delta t)$ was read out, and the radial velocity $v=u_{e d} / \Delta t$ was determined. Then, taking into account the pressure in the chamber $\mathrm{P}$, the geometrical parameters $B, b, r_{0}$ and $r_{e}$, and permeability $k$ and the liquid viscosity $\eta$, assumed in the simulations, the parameter $M$ could be determined from Eq. (22). The procedure was repeated for all combinations of values of stiffness of subcutaneous tissue and skin and subcutaneous tissue permeability, as given in Table 1, and the results for parameter $M$ along with the average values for a given combination of stiffness of the considered layers are summarized in Table 4 . The results show that the average values of the parameter $M$ range from 0.16 to 0.38 , and the average found from all combinations of the considered parameters is approximately 0.3 . Assuming this value of $M$ in Eq. (22) means that the procedure can be used to obtain a rough approximation of permeability. Despite this, taking into account the large range of possible values of the permeability of subcutaneous tissues (e.g., according to [9] from $10^{-16}$ to $10^{-9} \mathrm{~m}^{2}$ ) and the fact that, in clinical practice, there are no effective methods for determining the permeability of subcutaneous tissues in in vivo tests, the proposed procedure may be diagnostically useful.

\section{Discussion}

Analytical formulas relating the shear modulus and permeability of a tissue to changes in the radius or radial velocity of the outer boundary of a limb, respectively, for a single cycle of loading in the chamber tests were proposed. The obtained formulas were formulated based on simple models of limb geometry and tissue deformation. Cylindrical limb geometry guarantees that a limited number of geometrical parameters must be known, and the final formulas have a straightforward algebraic form. Complex dependences of stress and strain fields on spatial coordinates and time in the two-phase medium do not need to be

Table 4. Calibration constant $M$ (Eq. (22)) determined for different stiffnesses of subcutaneous tissue and skin and permeability of subcutaneous tissue (data for $50 \mathrm{mmHg}$ )

\begin{tabular}{|c|c|c|c|}
\cline { 2 - 4 } \multicolumn{1}{c|}{} & \multicolumn{3}{c|}{ Young's modulus of subcutaneous tissue used in simulations [kPa] } \\
\cline { 2 - 4 } \multicolumn{1}{c|}{} & 12.5 & 25 & 50 \\
\hline Young's modulus of skin & \multicolumn{3}{c|}{ Calibration constants $M$} \\
used in simulations [MPa] & for permeability $5 / 15 / 45 \times 10^{-14} \mathrm{~m}^{2}$ and the average value of $M$ \\
\hline \multirow{2}{*}{0.25} & $0.18 / 0.18 / 0.11$ & $0.31 / 0.25 / 0.17$ & $0.4 / 028 / 0.18$ \\
& av. 0.16 & av. 0.25 & $0.45 / 0.33 / 0.22$ \\
\hline \multirow{2}{*}{0.5} & $0.45 / 0.4 / 0.28$ & $0.45 / 0.36 / 0.28$ & av. 0.33 \\
\hline
\end{tabular}


considered, including the prestress of tissue matrix as a result of the undrained deformation stage and residual deformation of tissue after unloading (the case of 2nd or 3rd loading periods). Instead, models in which the outer boundary of the loaded part of the limb shrinks homogeneously (undrained stage) or is constant over time and $z$ coordinate (drained stage) were derived.

The proposed estimation methods were then applied to a sample of clinical data shown in Fig. 2. Since some parameters present in the derived estimation formulas were not determined in the chamber inflation-deflation test, additional measurements or assumptions were necessary. In particular, the initial radius $R_{e}$ (circumference $C_{0}=2 \pi R_{e}$ ) of the limb and the thickness of the oedematous tissue $R_{e}-R_{0}$ were not known from the chamber tests. The former parameter should be measured for the cross-section in which the strain gauge is located, whereas the tissue thickness should represent an average value under the test chamber and can be found by a comparison of oedematous and healthy limbs. According to clinical observations [17], the distance from the compressed subcutaneous tissue to the place where the pore pressure is dissipated, $b$, amounts to approximately three centimetres. Based on the recording of the chamber test results, shown in Fig. 2, the change in circumference for the undrained deformation $\Delta C_{u}$ and the increment of circumference as the result of the drained deformation $\Delta C_{d}$ can be found for the first loading cycle (Table 5), while the initial values of radius $R_{e}$ and $R_{0}$ are approximately equal to 70 and $55 \mathrm{~mm}$.

Using Eqs. (5), (21), the axial stretch ratio $\chi$ and shear modulus $\mu$ were estimated. Calculating radial velocity $v$, the permeability $k$ was determined using Eq. (22), assuming $M=0.3$ (Table 5). The values of the estimated shear modulus $\mu=15.9 \mathrm{kPa}$ and permeability $k=4.0 \times 10^{-14} \mathrm{~m}^{2}$ were within the limits of values used for simulations, which were used to determine the calibration constant $M$.

Table 5. The shear modulus and permeability ( $\mu$ and $k$ ) estimated from clinical results shown in Fig. 2 for loading cycle 50 [mmHg]

\begin{tabular}{|c|c||c|c|}
\hline Parameter & Value & Parameter & Value \\
\hline$\Delta C_{u}[\mathrm{~mm}]$ & 9 & $\Delta C_{d}[\mathrm{~mm}]$ & 1.8 \\
\hline$\chi[-] /$ Eq. (5) & 1.12 & $v=\frac{d r_{e}}{d t}[\mathrm{~m} / \mathrm{s}]$ & $1.6 \times 10^{-6}$ \\
\hline$\mu[\mathrm{kPa}] /$ Eq. (21) & 15.9 & $k\left[\mathrm{~m}^{2}\right] /$ Eq. $(22)$ & $4.0 \times 10^{-14}$ \\
\hline
\end{tabular}

The typical chamber test results, as shown in Fig. 2, are composed of a few inflation-deflation cycles, and for each cycle, fast deformation and slow creep are observed within the loading period. In some cases, however, the clinical results of the chamber test do not follow the systematic outflow of interstitial fluid from the tissue at each loading level because a certain pressure threshold must be exceeded to open for interstitial fluid flow [21]. For such cases, the permeability should be estimated for the cycle with the lowest load during which creep occurs (the linear model is assumed). If free interstitial fluid drainage does not appear in any cycle (when the contribution of free fluid is low due to the presence of stiff mature collagen bundles and/or fat globules [23]), the permeability of tissue cannot be determined.

The results of the estimation of hydromechanical parameters of tissues presented above were obtained in in vivo measurements for the subcutaneous edema tissue of the lower limb. It is difficult to find results in the literature for the set of conditions indicated. Among the available in vivo data for the subcutaneous tissue of the lower limb without oedema (for healthy tissue) one can find data obtained with the indenter method using the ultrasound technique [24]. The measured range of the Young's modulus was $60-180 \mathrm{kPa}$. With the assumption of incompressible tissue, it gave the shear modulus of 20-60 kPa. On the other hand, the use of tools such as MRI, ultrasound elastography together with FEM modelling and optimisation technique of identification made it possible in the case of the fatty subcutaneous tissue of the lower limb to find the average value of Young's modulus of $11-32 \mathrm{kPa}$ [8], which, assuming incompressibility, gives shear modulus of $3.66-10.66 \mathrm{kPa}$. Thus, the value of the shear modulus $\mu=15.9 \mathrm{kPa}$ obtained in this study for oedema tissue is slightly higher than the average modulus for adipose tissue reported in [8] and lower than the result presented in [24]. These differences may be justified by the fact that the edematous tissue may be stiffer than adipose tissue due to the developing network of fibers of the extra-cellular matrix. On the other hand, the indentation method used in [24] overestimates the rigidity of the subcutaneous tissue without separating the contributions of subcutaneous tissue and skin.

It is very difficult to find information on the permeability of the human lower limb subcutaneous tissue with oedema measured in vivo in the available literature. The value determined from chamber test end squeezing model $k=4.0 \times 10^{-14} \mathrm{~m}^{2}$ is within the range given for animal tissues without oedema and with artificially induced oedema that can range from $10^{-16}$ to $10^{-9} \mathrm{~m}^{2}$ [9]. The prerequisite for determining tissue permeability is the use of a tissue model 
as a fluid-filled porous material for interpretation of the measurement results, and this approach is still rarely used.

A review of the soft tissue property identification techniques developed so far can be found in [1] and [25]. Advanced techniques are laboratory methods that employ MRI, ultrasonic elastography and advanced 3D integration and inverse techniques, and are difficult to apply in typical clinical settings. Their advantage is the fact that they focus on taking the specific geometry and mechanical properties of the individual structure of the examined people into account. Another group of methods for testing the properties of soft tissues are indentation methods, which are relatively simple in terms of equipment and models used, but give information about local properties and usually ignore the presence of skin and the twophase nature of edema tissue in interpretation. The local properties do not need to be representative, and the omission of the biphasic nature of the subcutaneous tissues does not allow permeability to be determined.

The proposed method, due to the measurements of the circumference or the mean radius of the limb, is inherently a method that gives information about the averaged properties of the tissue for the limb fragment under the chamber. No more advanced hardware is required and there is no need to solve the complex inverse problem for real geometry. This gives a chance to transfer the proposed method to wider clinical practice. Due to the fact that the tissue results from beneath the chamber are collected from a relatively large area of tissue, they are not very sensitive to local inhomogeneities, and the skin effect is not as significant as in the case of indenter methods.

Certain limitations on the acquisition of appropriate clinical data from chamber tests result from the fact that the plethysmography method used to measure changes in limb circumference requires relatively expensive and, in some cases, problematic equipment. The sources of technical problems are mostly very sensitive plethysmographic strain gauges, which are prone to erroneous alignment in the test chamber, especially for irregular geometries. Further work is needed to confirm the clinical usefulness or specify the limitations of the proposed identification methods related to complex geometry or inhomogenous tissue properties. It is also worth making the effort to combine a diagnostic method using a chamber test and a therapeutic method of intermittent pneumatic compression in one device, which could constitute a new type of the so-called the theranostic method. Such a technique available at home would provide patients with new opportunities to observe the effects of treatment and perhaps its optimisation.

\section{Conclusions}

A simple in vivo method to estimate the hydromechanical properties of lymphedematous tissue: shear modulus and permeability was proposed. Analysis of the results of 3D numerical simulations performed for a complete two-phase approach confirmed that the fundamental assumptions of the proposed models to estimate stiffness and permeability are satisfied, particularly separation of the process into undrained and drained stages, the simple form of deformation of the outer boundary for the loaded part of the limb (independence of radial displacement and velocity on the axial coordinate in the first and second deformation stage, respectively), and the distribution of pore pressure in the model to estimate permeability. Finite element simulations were also used to verify the equation to estimate the shear modulus and calibrate the formula for permeability.

\section{Acknowledgements}

This work was partially supported by the National Science Center (grant UMO-2013/11/B/ST8/03589) and the National Center for Research and Development (grant PBS3/B9/46/2015). The authors acknowledge the help of Prof K. Zingerman in derivation of the undrained large deformation model of tissue (Section 2.2).

\section{References}

[1] Avril S., Evans S., (eds.) Material Parameter Identification and Inverse Problems in Soft Tissue Biomechanics, Springer Int., 2017.

[2] Boyer G., Pailler Matttei C., Molimard J., Pericoi M., LaQuieze S., Zahouani H., Non contact method for in vivo assessment of skin mechanical properties for assessing effect of ageing, Medical Eng. Phys., 2012, 34, 172-178.

[3] CASLEY-SMITH J.R., The fine structure and functioning of tissue channels and lymphatics, Lymphology, 1980, 12, 177-183.

[4] Chan W.-H., Huang Y.-L., Lin C., Lin C.-Y., Cheng M.-H., CHU S.-Y., Acoustic radiation force impulse elastography: Tissue stiffness measurement in limb lymphedema, Radiology, 2018, 289, 759-765.

[5] Cowin S,C., Doty S.B., Tissue mechanics, Springer Science, 2007.

[6] Dragan S.Ł., Kuropka P., Kulej M., Gabryś P., Nikodem A., Changes in the mechanical properties of femoral cartilage tissue in advanced osteoarthritis, Acta Bioeng. Biomech., 2020, 22 (1), 143-152.

[7] Feldman J.L., Stout N.L., Wanchai A., Stewart B.R., CORNIER J.N., ARMER J.M., Intermittent pneumatic compression therapy: A systematic review, Lymphology, 2012, 45, 13-25. 
[8] Frauziols F., Molimard J., Navarro L., Badel P., Viallon M., Testa R., Avril S., Prediction of the biomechanical effects of compression therapy by finite element modeling and ultrasound elastography, IEEE Trans. Biomedical Eng., 2015, 62, 1011-1019.

[9] Guyton C., Scheel K., Murphree D., Interstitial fluid pressure: III. Its effect on resistance to tissue fluid mobility, Circulation Research, 1966, 19, 412-419.

[10] Hara H., Mihara M., Comparison of two methods, the sponge method and Young's modulus, for evaluating stiffness of skin or subcutaneous tissue in the extremities of patients with lymphedema: A pilot study, Lymphat. Res. Biol., 2018, 16, 464-470.

[11] Iivarinen J.T., Korhonen R.K., JulKunen P., JuRvelin J.S., Experimental and computational analysis of soft tissue stiffness in forearm using a manual indentation device, Med. Eng. Phys., 2011, 33, 1245-1253.

[12] IIVARINEN J.T., Korhonen R.K., JuLKUnEN P., JuRVELIN J.S., Experimental and computational analysis of soft tissue mechanical response under negative pressure forearm, Skin. Res. Techn., 2013, 19, e356-e365.

[13] KaczmareK M., Olszewski W.L., NowaK J., ZalesKa M., The hydromechanics of edema fluid in lymphedematous lower limb during intermittent pneumatic compression, Lymphat. Res. Biol., 2015, 13, 260-267.

[14] KaczmareK M., Subramaniam R., NefF S., The Hydro-Mechanics of Hydrocephalus: Steady State Solutions for Cylindrical Geometry, Bull Math. Biol., 1997, 59, 295-323.

[15] Li C., BorJa R.J., Regueiro R.A., Dynamics of porous media at finite strain, Comput. Methods Appl. Mech. Eng., 2004, 193, 3837-3870.

[16] LORET B., SiMOES F.M.F., Biomechanical aspects of soft tissues, CRC Press, 2017.

[17] Olszewski W.L., Jain P., Ambujam G., ZalesKa M., CAKALA M., GRADALSKI T., Tissue fluid pressure and flow in the subcutaneous tissue in lymphedema-hints for manual and pneumatic compression, Phlebolymphology, 2010, 17, 144-150.

[18] Pailler-Mattei C., Bec S., Zahouani H., In vivo measurements of the elastic mechanical properties of human skin by indentation tests, Med. Eng. Phys., 2008, 30, 599-606.

[19] Rockson S.G., Lymphedema, Am. J. Med., 2001, 110, 288-295.

[20] WIIG H., SWARTZ M.A., Interstitial fluid and lymph formation and transport: Physiological regulation and roles in inflammation and cancer, Physiol. Rev., 2012, 92, 1005-1060.

[21] Zaleska M., OlsZEWSKI W.L., DuRliK M., The effectiveness of intermittent pneumatic compression in long-term therapy of lymphedema of lower limb, Lymphat. Res. Biol., 2014, 12, 103-109.

[22] Zaleska M., Olszewski W.L., Cakala M., Cwikla J., BUTLEWSKI T., Intermittent pneumatic compression enhaces formation of edema tissue fluid channels in lymphedema of lower limbs, Lymphat. Res. Biol., 2015, 13, 146-153.

[23] Zaleska M., Olszewski W.L., Durlik M., Kaczmarek M., A Novel Clinical Test for Setting Intermittent Pneumatic Compression Parameters Based on Edema Fluid Hydromechanics in the Lymphedematous Calf, Lymphat. Res. Biol., 2015, 13, 208-214.

[24] ZHENG Y.-P., MAK A.F.T., LuE B., Objective assessment of limb tissue elasticity: development of a manual indentation procedure, J. Rehab. Res. and Dev., 1999, 36, 71-85.

[25] ZHENG Y.-P., HuANG Y.-P., Measurement of soft tissue elasticity in vivo, CRC Press, 2016.

[26] Zingerman K.M., LeVIn V.A., Some qualitative effects in the exact solutions of the Lame problem for large deformations, J. Appl. Math. Mech., 2012, 76, 205-219.

[27] ŻMudzińsKa M., Inglot M., ZALESKA-Dorobisz U., JANKOWSKi L., ŚWIĄTEK-NAJWER E., The assessment of the applicability of shear wave elastography in modelling of the mechanical parameters of the liver, Acta Bioeng. Biom., 2018, 20 (4), 59-64. 